\title{
ASSESSMENT FOR A CONDITION USING TERRESTRIAL LIDAR DATA
}

\author{
J. S. Park ${ }^{1, *}$, K. S. Lee ${ }^{1}$, S. Kim ${ }^{1}$ \\ ${ }^{1}$ Disaster Scientific Investigation Division, National Disaster Management Research Institute, Ulsan, Korea - (jesung0225, 39lks, \\ sskim)@korea.kr
}

Commission VI, WG VI/4

KEY WORDS: Pothole, Road condition, Terrestrial LiDAR, Trend analysis

\begin{abstract}
:
The pothole is called a road breakage where the surface of road is locally recessed. The conventional vehicle-mounted pothole detection sensor acquires data only when the vehicle directly contacts the pothole and moreover, the acquired data is limited to show the position information of the pothole based on the low-precision GPS. We have, therefore, studied the assessment methods for the road condition using the terrestrial LiDAR data to complement and improve the current methodology. In this study we subtract the low-resolution raster data from the high-resolution raster one that are taken from the terrestrial LiDAR, which is similar to the trend analysis. We assessed the proposed method using the manholes, which we assumed as potholes. It also showed that our method can improve a variety of perspectives such as time consuming, space extension and quantitative interpretation. As a result, we could expect the proposed method will be effectively used in the field of road management.
\end{abstract}

\section{INTRODUCTION}

The pothole is called a road breakage where the surface of road is locally recessed (Miller \& Bellinger, 2003). The formation of potholes in winter in the presence of water, is quite associated with freeze thaw cycles (Hershfield, 1979). Korea is in the same situation, a pothole is often occurred with the effect of the deicing chemicals, which prevent snow in road. These potholes having a major impact on the vehicle. In particular, the potholes have a deadly effect on motorcycles. As indicated by the review report 'Road Accidents in India (2017)' has shown, the potholerelated accidents had claimed 2,597 lives in 2017 (Saha et al., 2018). In response, the Korean government operated a road pavement improvement $\mathrm{T} / \mathrm{F}$ team in 2013 to improve the porthole that occurs on the road. Also, many researchers have tried to detect the pothole effectively (Mednis et al., 2011; Chen et al., 2013; Koch\&Brilakis, 2011; Ryu et al., 2015). For the currently developed pothole sensor, it is detected when the sensor comes into direct contact with the pothole. It is hard to repeated runs on the same track with consistently same conditions (Strazdins et al., 2011). Moreover, the acquired data is limited to show the position information of the pothole based on the low-precision GPS. Most of the research is confined to the pothole. In 2017, many lives were lost in the 2017 by the rollover accident of engine oil transport vehicle in Changwon, Korea. There was no the pothole in the accident area, but the condition and complex environment of the road surface was very poor. To prevent the similar accident, the Korean government is planning a road-related disaster risk assessment in the second half of 2019. We have, therefore, studied the assessment methods for the road condition using the terrestrial LiDAR (Light Detection And Ranging) data to complement and improve the current methodology. Further, the study is a preliminary study to prepare for the government-initiated planning investigation. Terrestrial LiDAR is a remote sensing technique that transmits a laser beam into the atmosphere and the backscattered light is detected (Kasler et al., 2010). With these advantages, LiDAR has been used in such fields as geophysics, engineerings, extraction building, Digital Surface Model (DSM) production and 3D city modelling (kreylos et al., 2008; Song et al., 2002). In this study, we subtract the lowresolution raster data from the high-resolution raster one that are taken from the terrestrial LiDAR, which is similar to the trend analysis. We assessed the proposed method using the manholes, which we assumed as potholes.

\section{CREATION ANALYTICAL DATA}

In this study, we analysed that the road was extracted from the terrestrial LiDAR data acquired in National Disaster Management Research Institute, Korea. Also, we acquired the point cloud data and created the analytical data using RIEGL company's software (Riscan Pro). This chapter introduces the process of producing analytical data from the initial data, such as noise elimination, road extraction and Triangulated Irregular Network (TIN) creation.

\subsection{Data Acquisition}

Data acquisition is proceeded by RiEGL company's terrestrial LiDAR(VZ-2000). The VZ-2000 acquires 400,000 point data per second, with an accuracy of $8 \mathrm{~mm}$ and a precision of $5 \mathrm{~mm}$. We operated the terrestrial LiDAR at a total of 16 points by a static survey, each of which matched the acquired data with each other. We operated the terrestrial LiDAR on a static survey method at a total of 16 positions. In addition, each acquired data was matched. We performed the data matching using the RiscanPro software based on the data of a single position that performed the absolute orientation. The ground control points (GCPs) were surveyed using Trimble's GNSS R10 during 4 hours per position to process the absolute orientation. The root mean square error (RMSE) for the coordinates of the GCPs were 0.076 . Also, the matching RMSE

\footnotetext{
* Corresponding author
} 
for the terrestrial LiDAR data in 16 position was 0.008 . The matched data is shown in figure 1.

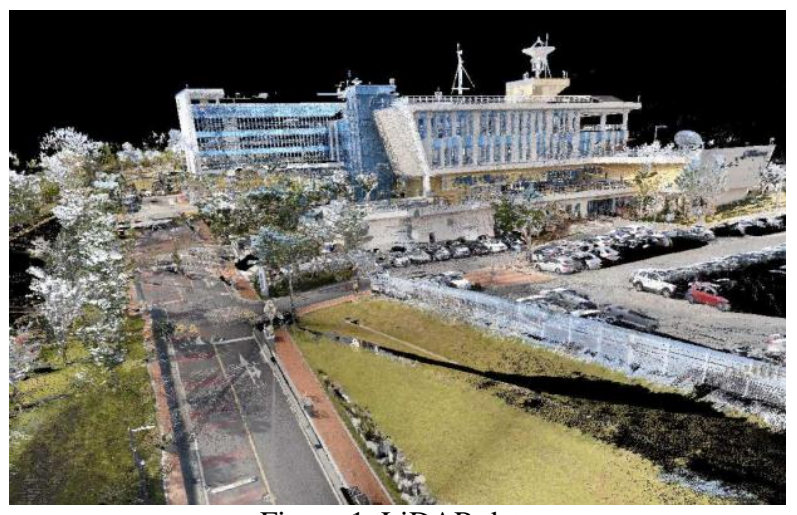

Figure 1. LiDAR data

\subsection{Generating Analysis Data}

We extracted only roads from the matched data to assess the condition of the road. In addition, we removed noise such as vehicles and road facilities from the extracted data. These modified data were made from two types of TIN data. The reason why data is made into TIN data is to interpolate the point cloud data because this data has the space of no data between each point. One data is high-resolution TIN data that can accurately represent the road surface, and the other data is lowresolution TIN data that normalize the road surface. The concepts of both data are shown in the figure 2 .

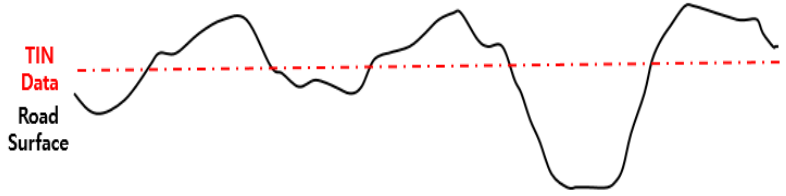

Figure 2. TIN data concept

In figure 2, TIN Data is low-resolution TIN data and the road surface is high-resolution TIN data. In case of low-resolution data, it is made as the assumption that the road surface is perfect.

\section{DATA ANALYSIS}

We conducted the road condition assessment and the proposed method assessment.

\subsection{Assessment for a Road Condition}

We subtracted the high-resolution data from the low-resolution data, before the road condition assessment. Data produced in this method can represent the road conditions by eliminating road slopes. In this data, the position at which the negative value is obtained is lower than the average ground, and the position which the positive value is higher than the average ground. In addition, because the road surface is not smooth, we defined which position is lower than the standard deviation value from the mean value as the position which abnormal symptoms occurred. The abnormal positions can be pothole. This concept is shown in the figure 3 .

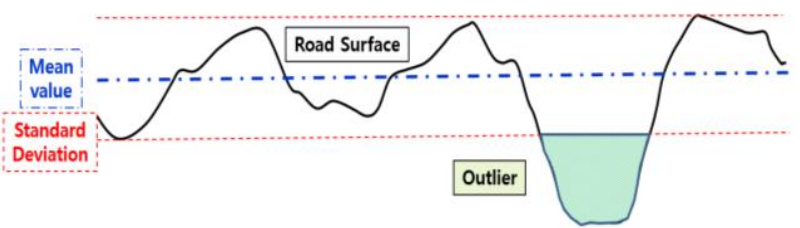

Figure 3. Road condition assessment concept

We are all of this data processing and analysis using ArcGIS software. The subtracted data in this method is shown in the figure 4 .

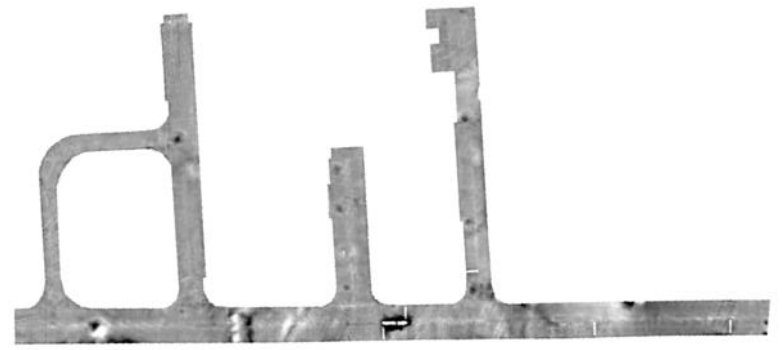

Figure 4. Subtracted data

The standard deviation of the subtracted data for the study road was $0.009 \mathrm{~m}$. The detected outlier data is shown in the figure 5 .

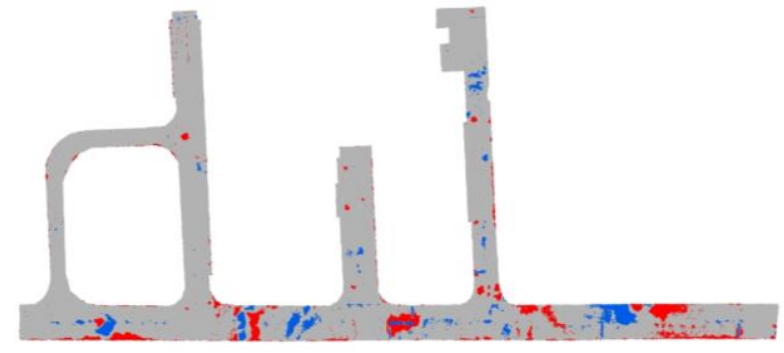

Figure 5. Detection outlier

In figure 5, the blue zone expresses the positions which are higher than the average ground and the red zone expresses the positions which are lower than average ground. We easily understand the condition of particular road though this data. The total area of the study road is $2,084 \mathrm{~m}^{2}$. Of these, the total area of the blue zone is $76 \mathrm{~m}^{2}$, which is about $3 \%$ of the total area, and the standard deviation is $0.02 \mathrm{~m}$. Also, the total area of the red zone is $105 \mathrm{~m}^{2}$, which is about $5 \%$ of the total area, and the standard deviation is $0.015 \mathrm{~m}$. The institute was established in 2015, and the road is good condition. However, we are difficult to assess the condition of the road as only this numerical value. Detailed evaluation takes into account both the area and standard deviation of the outlier, and requires criteria for the assessment. Therefore, we will find the assessment criteria of road condition through assessment for the many roads, when perform the government planning investigation in this year.

\subsection{Assessment for Method}

We analysed the manholes assuming the potholes to assess our proposed method. First, we conducted a Network-RTK surveying to obtain location information about manholes using GPS. GPS measurements were made in 10 seconds, and the observed manholes were 16 positions. We built the manhole data through buffer tools in ArcGIS using measure position information of the manholes. The actual size of the manhole is 
$700 \mathrm{~mm}$ in diameter, and the resolution of the raster data is 1 $\mathrm{mm}$. Figure 6 is the sample image which show a raster data for the extracted manhole.

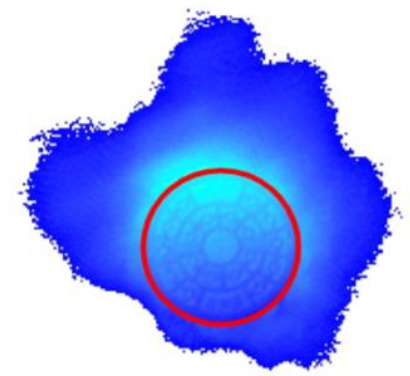

Figure 6. Sample image (manhole)

The results of extracting manhole data from the subtracted data are shown in Table1.

\begin{tabular}{|c|c|c|}
\hline Manhole & \multicolumn{2}{|c|}{ Analysis } \\
\cline { 2 - 3 } ID & Area $\left(\mathrm{mm}^{2}\right)$ & Ratio (\%) \\
\hline 1 & 62 & 1.61 \\
2 & 1063 & 27.62 \\
3 & 0 & 0 \\
4 & 0 & 0 \\
5 & 3824 & 99.36 \\
6 & 2767 & 71.90 \\
7 & 3802 & 98.79 \\
8 & 1463 & 38.02 \\
9 & 2613 & 67.90 \\
10 & 802 & 20.84 \\
11 & 3623 & 94.14 \\
12 & 795 & 20.66 \\
13 & 1556 & 40.43 \\
14 & 1079 & 28.04 \\
15 & 3828 & 99.47 \\
16 & 0 & 0 \\
\hline
\end{tabular}

Table 1. Result of manhole analysis

In Table 1, the ratio is the percentage of the detected area in the actual area of the manhole. The actual area of the manhole used in this study is $3848.45 \mathrm{~m}^{2}$. From the data analysis, we could see that the manholes were detailed. As this result, we could know that the slope value of each different position is removed and the road surface is flattened when subtracted the high-resolution TIN data from the low-resolution TIN data. Thus, our proposed method in this study can represent the sufficiently detailed road surface. However, as a result of area analysis, the accuracy of $44.3 \%$ was verified, and this value is not accurate. This is the error of our assumption that manholes are lower than the road surface.

\section{CONCLUSION}

We have studied the assessment methods for the road condition using the terrestrial LiDAR data to complement and improve the current methodology. Further, the study is a preliminary study to prepare for the government-initiated planning investigation. To achieve our goal, we conducted the road condition assessment and the proposed method assessment using the data of terrestrial LiDAR. We subtracted the highresolution data from the low-resolution data, before the road condition assessment. Data produced in this method can represent the road conditions by eliminating road slopes. As a result of analysis, we easily understand the condition of particular road though the produced data. In addition, we performed the numerical analysis using the data. However, we are difficult to assess the condition of the road as the numerical value in this study because we don't have the criteria for assessment. Therefore, we will find the assessment criteria of road condition through assessment for the many roads, when perform the government planning investigation in this year. However, the results of this study will be useful in assessing the construction roads ahead of completion, such as highways and national roads, or analysing the priority of the maintenance for the roads. We analysed the manholes assuming the potholes to assess our proposed method. Through assess our method, our proposed data processing in this study can represent the sufficiently detailed road surface. However the accuracy of $44.3 \%$ was verified, and it is not accurate. This is the error of our assumption that manholes are always lower than the road surface. As a result, we require more study for criteria of road condition and assessment for method. However, our proposed methodology showed that can improve a variety of perspectives such as time consuming, space extension and quantitative interpretation. Further, if we complement the criteria of road condition and the assessment method of our proposed methodology, we expect the proposed method will be effectively used in the field of road and disaster management.

\section{ACKNOWLEDGEMENTS}

This research outputs are part of the project "Development of forensic techniques for Disaster Scientific Investigation", which is supported by the NDMI (National Disaster Management Research Institute) under the project number NDMI-MA-201905-01. The authors would like to thank the financial support of the NDMI.

\section{REFERENCES}

Chem, K., Lu, M., Tan, G., Wu, J., 2013. CRSM: Crowdsourcing based road surface monitoring, Proc. IEEE Int. Conf. High Perform, Comput. Commun., 2151-2158. doi.org/10.109/HACC.and.EUC.2013.308.

Hershfield, D. M., 1974. Freeze-Thaw Cycles, Potholes, and the Winter of 1977-78. Journal of Applied Meteorology, 18, 10031007.

Kasler, Y., Rahm, S., Simmet, R., 2010, Wake Measurement of a Multi-MW Wind Turbine with Coherent Long-Range Pulsed Doppler Wind Lidar, J. Atmos. Oceanic Technol., 27, 15291532, doi.org/10.1175/2010JTECHA1483.1.

Koch, C., Brilakis, I., 2011. Pothele detection in asphalt pavement images, Advanced Engineering Informatics, 25(3), 507-515.doi.org/10.1016/j.aei.2011.01.002.

Kreylos, O., Bawden, G. W., Kellogg, L. H., 2008, Immersive visualization and analysis of LiDAR data, International Symposium on Visual Computing, Advances in Visual Computing, 846-855.

Mednis, A., Strazdins, G., Zvidedris, R., Kanonitsm, G., Selavo, L., 2011. Real time pothole detection using android smartphones with accelerometers. In IEEE DCOSS. doi.org/10.1109/DCOSS.2011.5982206.

Miller, J. S., Bellinger, W. Y., 2003: Distress identification manual for the long-term pavement performance program.

Ryu, S. K., Kim, T., Kim, Y. R., 2015, Image-Based Pothole Detection System for ITS Service and Road Management 
System. Mathematical Problems in Engineering, 501, 968361. doi.org/10.1155/2015/968361

Saha, S. K., Batt, R., Gavankar, A., Singh, A. P., 2018. A Review Paper on Existing Pothole Detection Methods, International Research Journal of Engineering and Technology(IRJET), 5(12), 566-568.

Song, J. H., Han, S. H., Yong, K. Y., Kim, Y. I., 2002, Assessing the possibility of land-cover classification using LiDAR intensity data. International Archives of Photogrammetry, Remote Sensing and Spatial Information Sciences, 34(3A), 259-262.

Strazdins, G., Mednis, A., Kannirs, G., Zviedris, R., Selavo, L., 2011. Towards vehicular sensor networks with android smartphones for road surface monitoring. In 2nd International Workshop on Networks of Cooperating Objects(CONET11), Electronic Proceeding of CPS Week, 11. www.researchgate.net/publication/235429698. 